\title{
A Design of Monitor Terminal for Real-time State of Electric Vehicle Based on Dual Buses
}

\author{
YAN Yu-lin ${ }^{1, a}$, LIU Chun-guang ${ }^{1, a}$, Liao Zi-li $1^{1 \text {, a }}$, HUANG Jie-yin ${ }^{2 \text {, a }}$ \\ ${ }^{1}$ Academy of Armored Force Engineering, Beijing, 100072, China \\ ${ }^{2}$ NO.447Factory Military Representative Office of Beijing Military Bureau, Inner Mongolia, Baotou \\ 014030, China \\ aemail: 530144848@qq.com
}

Keywords: CanOe, Monitoring Terminal, CAN bus, FlexRay bus

\begin{abstract}
Aiming at the numbers of system state parameters and high real-time requirements of real vehicle test, in order to monitor and record various status indicators in actual operation of Multi-Wheel-Independent-Drive(M-WID) Electric Vehicle with in-wheel motor, a real-time monitor recording terminal equipment was developed with CanOe, which is based on CAN bus and FlexRay bus. The practical results showed that the terminal achieved the collecting, displaying, fault alarming and saving of the communication signals in the actual operation of the vehicle in real-time. The monitoring terminal is stable and reliable, which provides a basis for further adjusting the control parameters of the vehicle and improving the control algorithm.
\end{abstract}

\section{Introduction}

Multi-Wheel-Independent-Drive(M-WID) Electric Vehicle with in-wheel motor is a complex system of electric vehicle which set electrical, control, mechanical and other disciplines as one, in order to get real time status information of the vehicle in the process of real vehicle test, the vehicle communications signals and control signals need to be collected for providing the basis of vehicle running attitude adjustment to the driver[1]; in order to analyze vehicle and system performance, improve energy management, it is necessary to monitor the running state parameters of the vehicle in real time and record the required state quantity for the following research. Therefore, it is needed to design and develop the vehicle real-time condition monitoring terminal, [2]. This paper analyzes the vehicle communication structure and definition, determines the state parameters need to be monitored and collected, designs the vehicle real-time state monitoring terminal based on CanOe bus software, and realized the effective monitoring of actual running state of vehicle[3].

\section{Vehicle Communication System Structure}

The communication system of Multi-Wheel-Independent-Drive (M-WID) electric Vehicle with in-wheel motor provides the information interaction platform among components, for the transmission of vehicle state information, control signal, and fault information. The main communication components includes low voltage distribution box, high voltage distribution box, engine control box, the central controller, battery manager, state collection box, brake control box, and four motor drives, among the components, real-time communication is established through the CAN bus and FlexRay bus network. The overall structure of the vehicle communication system is shown in figure 1. 


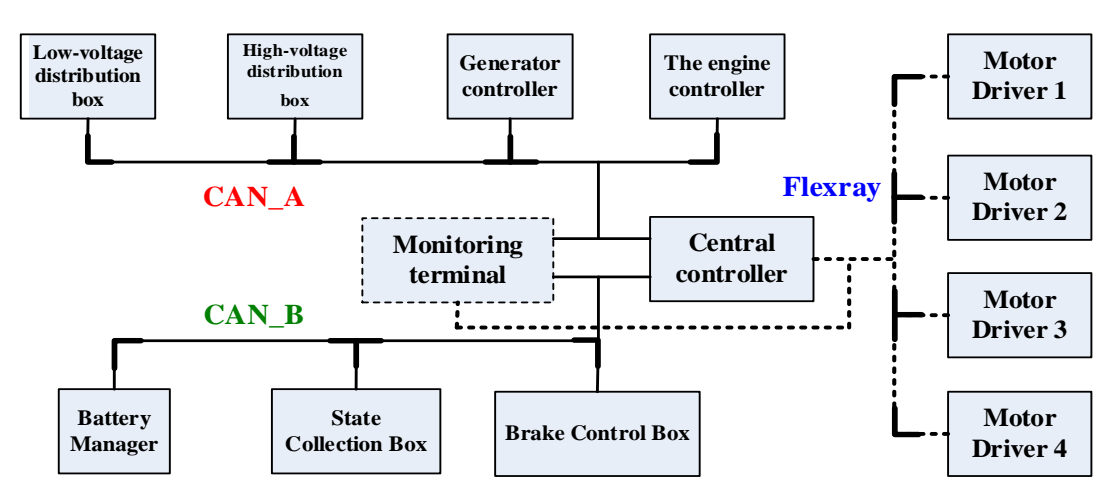

Fig. 1 Bus Network Structure

Vehicle bus network includes CAN network and Flexray network. The CAN network is divided into two mutually independent networks according to the situation of information interaction: CAN_A network and CAN_B network.

The central controller, low voltage distribution box, engine electric control box, high voltage power distribution box, generator controller, power DC/DC, and display control terminal constitute CAN_A network, state collection box and brake controller constitute CAN_B network, Flexray bus is adopted between the central controller and the drive motor frequency converter.

In real vehicle experiment ,the monitoring terminal needs to realize the function mainly includes the following three parts: Firstly, displaying and monitoring the driver's manipulation signals, high and low voltage distribution instructions, brake signals, steering signal, high voltage pre charge indicator, and low voltage power distribution indication, etc. in real-time. Secondly, displaying and monitoring a variety of vehicle status informations, including speed, yawing angular velocity, battery SOC, bus voltage, motor torque, DCDC working state and fault information, etc. in real-time. Lastly, to alarm when the data exceeds the threshold value and to store the record data for calling later.

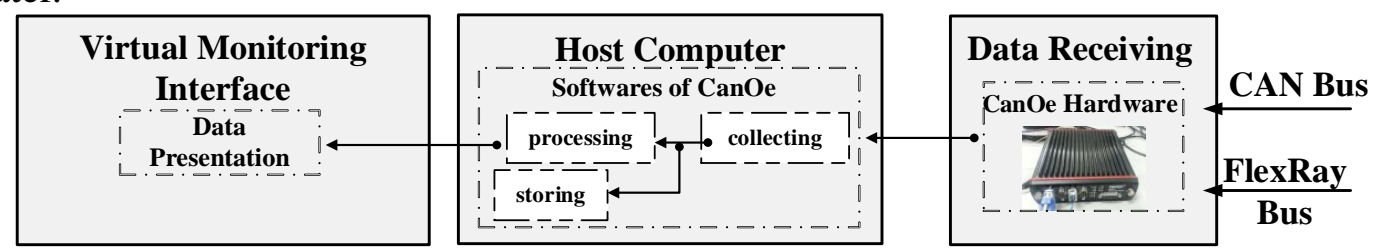

Fig. 2 structure of monitoring terminal system

To achieve the functions above, this paper designed a monitoring terminal as shown in figure 2, including three parts: CanOe hardware receiving data terminal, monitoring terminal PC, and the virtual monitoring interface.

The data message collected by CanOe hardware box from the bus was transferred to the host computer. According to bus communication protocol, the host computer analyzed and present the data message through the virtual monitoring interface with the CanOe development kit to, and stored desired state information to the host computer in the form of text file.

\section{Bus Communication Protocol Design}

In order to ensure that the communication between nodes is accurate and efficient, the communication node ID and the bus communication protocol between CAN network and FlexRay network have been deployed.

Design Of CAN Bus Communication Protocol. From Figure 1, the CAN bus is articulated by many parts, the transmission data is large, and the CanOe need to collect and parse transmission data from the CAN bus directly.

To the rational use of bus bandwidth, reduce bus redundancy information, improve the bus channel utilization and system reliability, unified coding the communication information on the CAN bus.

Bus control system adopts CAN2.0B extended frame format, according to the PGN code and the extension of the 29 bit identifier (ID) definition, combined with the communication equipment 
priority requirements, distribute A and B network communications equipment ID, as shown in Table 1 .

Table 1 Distribution of device ID

\begin{tabular}{ccc}
\hline Net & Nodes & ID (Hex) \\
\hline & Central Controller (Main) & 0x03 \\
\cline { 2 - 3 } & Central Controller (Slave) & 0x48 \\
\cline { 2 - 3 } CAN_A & Engine Controller & 0x00 \\
\cline { 2 - 3 } & High-voltage Distribution & 0x0F \\
& Box & 0x1C \\
\cline { 2 - 3 } & Generator Controller & 0x45 \\
\cline { 2 - 3 } & Low-voltage Distribution & Box \\
\hline & Central Controller & 0x05 \\
\cline { 2 - 3 } CAN_B & Brake Controller Box & 0x38 \\
\cline { 2 - 3 } & Battery Manager & 0x3F \\
\cline { 2 - 3 } & State Collection Box &
\end{tabular}

Defined by the CAN bus data frame format, a single data frame can transmit up to 8 bytes of data, and each node on the bus transmits data with different data volume, in order to avoid the increase in the number of e-mail address, make full use of the data frame length, defining data in accordance with the definition of data bits in the actual encoding, namely a byte of data CAN be multiple information fusion, no longer alone represent the actual information. To send a high voltage distribution box node data as an example, the message described as high-pressure tank sent to the central controller, refresh rate for the trigger mode, asked by the central controller, reply within $20 \mathrm{~ms}$, specific definition as shown in table 2 and table 3 :

Table 2 ID Definition of High-voltage Distribution Box

\begin{tabular}{|c|c|c|c|c|c|c|c|}
\hline \multirow[b]{2}{*}{ PGN } & \multicolumn{5}{|c|}{ ID:0x0C72030F } & \multirow[b]{2}{*}{$\begin{array}{c}\text { Refresh } \\
\text { Rate }\end{array}$} & \multirow{2}{*}{$\begin{array}{l}\text { Messa } \\
\text { ge } \\
\text { Length }\end{array}$} \\
\hline & Priority & $\begin{array}{l}\text { Rema } \\
\text { in }\end{array}$ & $\mathrm{PF}$ & PS/DA & SA & & \\
\hline 0x7200 & $0 \times 3$ & 0x0 & $\begin{array}{l}0 \times 7 \\
2\end{array}$ & 0x03 & $0 \mathrm{x} 0 \mathrm{~F}$ & Trigger & 8 Byte \\
\hline
\end{tabular}

Table 3 Message Content of High-voltage Distribution Box

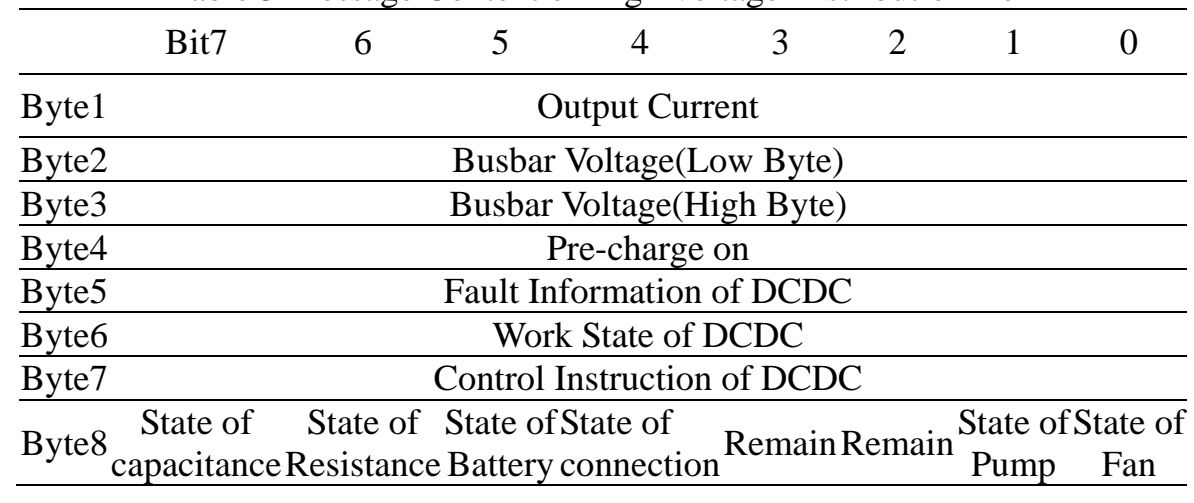

For more than a single data byte of data information, divide it into two parts in accordance with the Motorola rules, and then put them in the high and low byte of the same message, low byte first and high byte last, as the bus voltage in table 3, as far as possible to ensure the correlation of data contained in the same node, which is convenient for the subsequent parsing.

Flexray Communication Protocol Design. Flexray network adopts bus type network structure, which is dual redundancy bus and divided into A bus and B bus, all nodes devices are connected with $\mathrm{A}$ bus and B bus. In order to ensure the redundancy, the A bus and the B bus cable are independent of each other and are not connected with each other, and the independent plugs and sockets are used between each channel bus cable and the node devices.

In the case of motor driver 1, the design of message transmission protocol is shown in Table 4. 
Table 4 FlexRay Communication Protocol of Motor Driver1(Part)

\begin{tabular}{|c|c|c|c|}
\hline & Cycle time: $5 \mathrm{~ms}$ & $0 \mathrm{X} 05$ & \\
\hline Byte & Data Range & $\begin{array}{c}\text { Signal } \\
\text { Description }\end{array}$ & Resolution \\
\hline $1-2$ & $0000-7530$ & DCU2 given torque 1 & $1 \mathrm{Nm}$ \\
\hline$\ldots$ & & & \\
\hline $20-21$ & Main controller send control words to motor 1: 1=effective & $\begin{array}{c}\text { Bit0:start 1 } \\
\text { Bit1:stop 1 } \\
\text { Bit2:forward 1 } \\
\text { Bit3:backward 1 } \\
\text { Bit4:traction 1 } \\
\text { Bit5:brake 1 } \\
\text { Bit6:neutral gear 1 } \\
\text { Bit7:busbar discharge 1 }\end{array}$ & \\
\hline
\end{tabular}

In order to reasonably allocate the message transmission time slot, define FlexRay communication data frame length is 32 bytes.

\section{Design And Development Of Real Time Monitoring Software}

Real-time monitoring software uses the Vector CanOe development platform, through the communication node configuration, data signal decoding, monitoring, recording and virtual monitoring interface design, completed the real-time monitoring software design.

Communication Node Configuration. After establishing the new project, using CANdb++ Editor and Fibex Explorer to establish CAN FlexRay network's configuration file, will generate the .dbc file and the .xml file into the original project, extract the corresponding node in databases to build the network, shown in Figure 3, Figure 4.

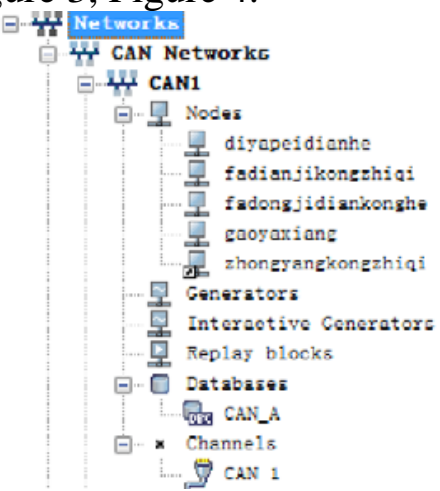

Figure 3 CAN_A network node

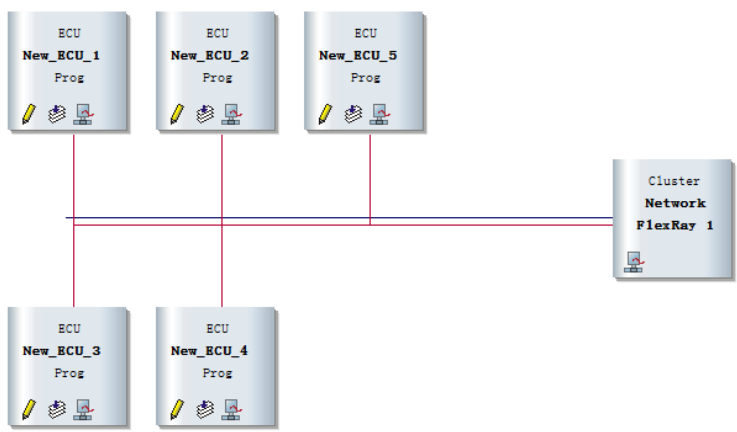

Figure 4 CAN-A network communication link

Data Decoding And Storage. According to the CAN network and FlexRay network communication protocol proposed in the last section, write messages and signals in CANdb++ Editor and Fibex Explorer respectively.

All messages in CAN network are independent add to Message, create a signal belonging to the message, set the signal length (bit), unit, gain, offset, ranking position of each signal in the message according to the high and low byte, finally classified messages to corresponding node by ID. Take the first data frame sent by the high pressure tank as an example, as shown in Figure 5 and figure 6 . 


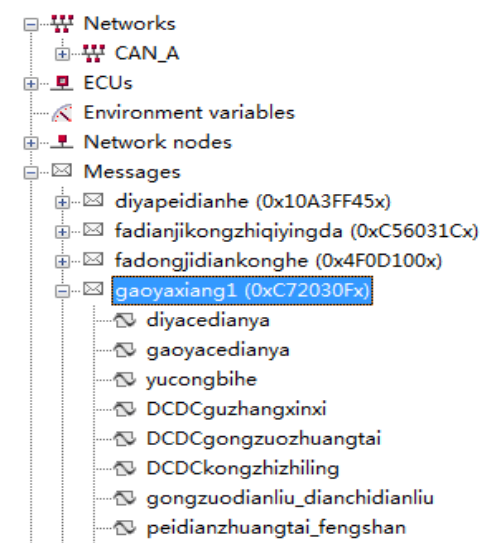

Figure 5 CAN network high pressure box node message preparation

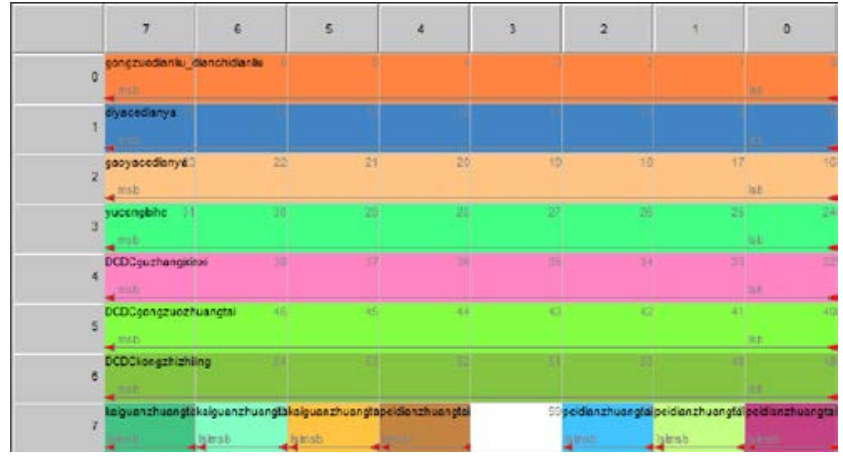

Figure 6 CAN network high voltage box signal sorting

The FlexRay network consists of A, B double redundancy channel, the message encoding is the same, add message sending from the node to the node add signal contained in the message. Different from the CAN network is the need to set the length and type of data frames. Take the motor drive 1 as an example, the specific settings as shown in Figure 7, 8.

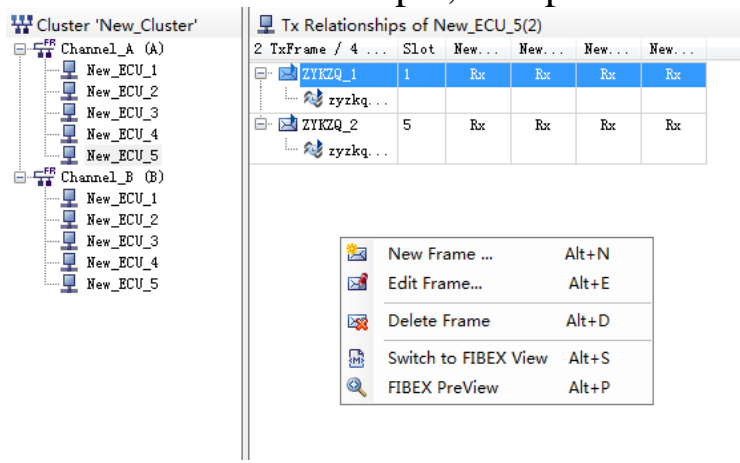

Figure 7 FlexRay network driver node message

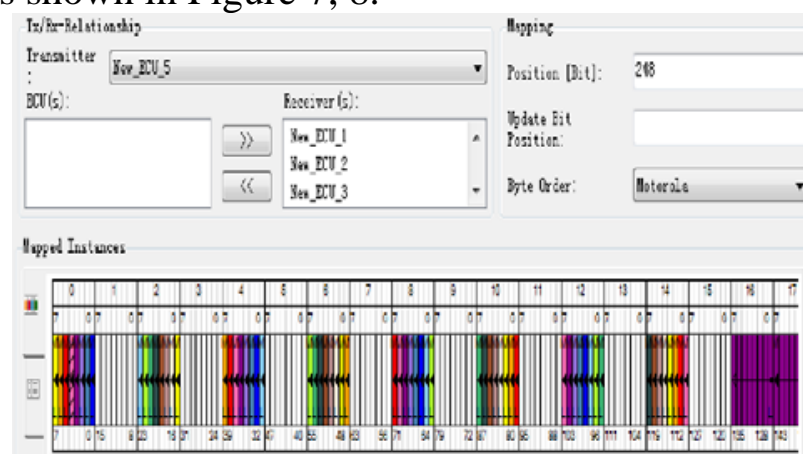

Figure 8 FlexRay network driver node signal

Vector CanOe can filter and save the data transferred by bus as .ASC files directly, the saved data is all signals corresponding to a ID number in the message, the specific data variables need to be treated with MATLAB later.

Channel Filter selects channels for all communication networks access to CanOe, embedded CFB module and Trigger module of the ID message in the channel selection, pre trigger time 0.05s, sampling frequency $100 \mathrm{~Hz}$, the buffer size of $1000 \mathrm{MS}$, as shown in figure 9 .

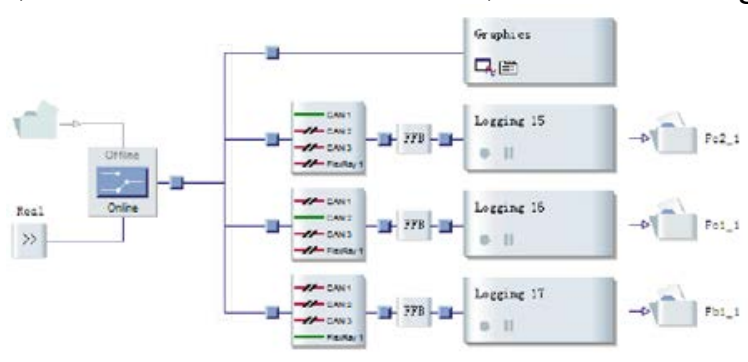

Figure 9 data storage settings

Monitoring Interface Design. The Panel Designer module provides a wealth of signal monitoring and external trigger tools. Set the monitoring screen resolution of $1440 * 700$, according to written communication protocol, dragging the signal which needs to be observed from the left side of the signal selection window into the signal display area directly.

Due to large amount of observation data, in order to beautify the interface, convenient observation, import the picture of the actual related parts from outside, transform the display of the enable signal or life signal into the picture; if the data in the monitoring process exceeds a threshold value are shown in red, it is Conducive to timely find the vehicle fault, design interface is shown in figure 10 . 


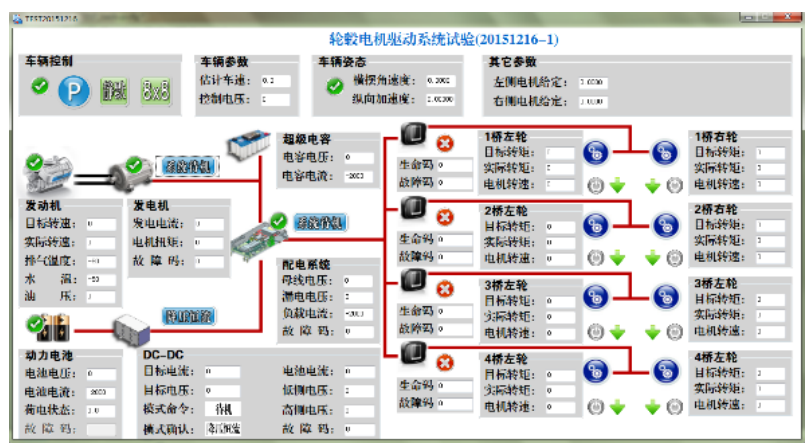

Fig. 10 terminal detection interface

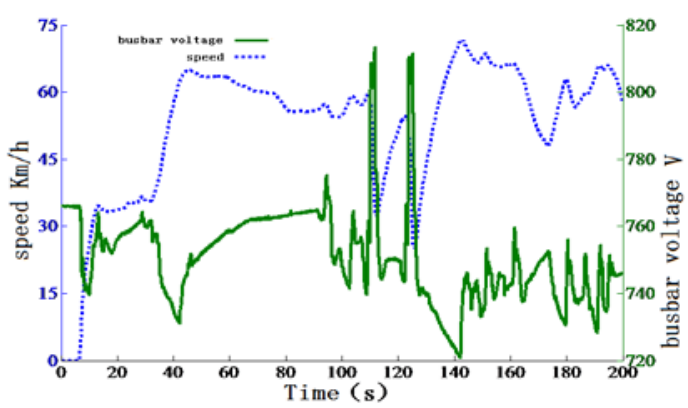

Fig. 11 speed and bus voltage

\section{Real Vehicle Test}

Figure 11 is the change curve of post treatment of the vehicle speed and bus voltage data variables which are collected during the operation of the real vehicle. $0 \sim 20 \mathrm{~s}$ is vehicle's starting stage, the accelerator pedal pressed down, the bus discharge drive motor; 20s to 40s, vehicle traveling at a constant speed, generator bus charging, voltage rise; in the 110 s and 120 s vehicle emergency braking, regenerative braking, bus voltage pump up the super capacitor charging, quickly fall back.

\section{Conclusion}

This paper design a data real-time monitoring terminal based on the CanOe bus development platform, realize the effective monitoring of real-time operating state of Multi-Wheel-Independent-Drive(M-WID) Electric Vehicle with in-wheel motor, data management is fast and effective, bus communication is safe and accurate, the monitoring interface is clear and beautiful, and it has achieved the expected design goal.

\section{References}

[1] Sun Fengchun, Zhang Chengning. Hybrid electric drive technology of the armored vehicles [M]. Beijing: National Defence Industry Press, 2008:56-84.

[2] Liu Qiuli, Liu Chunguang, Yuan Dong. Design of electricity transmission armored vehicle line display control terminal [J]. Automation \& Instrumentation, 2011(3):50-53.

[3] Zeng Qinghan, Liu Chunguang, Zhang Yang. Design of monitor terminal for real-time simulation system of electric-driven armored vehicle[J]. Journal of Computer Applications. 2012,32(s2):261-263.

[4] Song Xiaoqing. The military vehicle integrated electronic system bus network[M].Beijing: National Defence Industry Press, 2010:102-106.

[5] Jin Zhenhua, Lu Qingchun, Wei Hongjun. Development of Vehicle CAN bus monitoring system based on virtual instrument[J]. Application of Electronic Technique, 2006(11):79-81 